\title{
A Study on Protective Reuse of Traditional Architecture in Lhasa
}

\author{
Qing Qin ${ }^{1, *}$, Yang Chen ${ }^{1}$, and Haiyue Zhang ${ }^{1}$ \\ ${ }^{1}$ School of Human Settlements and Civil Engineering, Xi'an Jiaotong University, Xi'an 710049, China
}

\begin{abstract}
Traditional Tibetan architecture in Lhasa has a unique position in the world. Under the special social background of economic development demand and tourism, it has gradually become a pillar industry, and how to rationally protect and reuse this traditional architecture has become an urgent problem that must be solved. Based on the traditional architectural patterns, regional characteristics and modern needs of Lhasa, this paper discusses the strategies for the protection and reuse of architectural heritage, the specific methods of reuse and their advantages and disadvantages, in order to provide a reference for the protection of traditional buildings and the inheritance of culture in Tibet.
\end{abstract}

\section{Introduction}

Traditional Tibetan architecture, as one of the world's most unique architectural types, has distinctive characteristics and occupies a very special position. Under the combined effect of the particularity of the natural environment caused by the snow plateau and the humanistic environment formed by the Tibetan people's religious beliefs, traditional Tibetan architecture has gradually formed its unique architectural system and design methods. Lhasa has been the political, economic and cultural center of Tibet since ancient times. The population of Lhasa accounts for about $27 \%$ of the total population of Tibet, and has the most representative and well-preserved Tibetan architecture. Therefore, this study on the protective reuse of traditional buildings in Lhasa can provide a theoretical basis and strategies for the protection of traditional buildings in Tibet.

\section{Overview of traditional architecture in Lhasa}

\subsection{The types and characteristics of traditional architecture in Lhasa}

Lhasa is located on the Tibetan plateau at an altitude of more than 3500 meters, forming a plateau temperate semi-arid monsoon climate, which is cold and dry in winter and cool and rainless in summer. Under the influence of this climatic condition, the unique flat-roofed building form with a wooden framework and mud and stone wall structure has developed in Lhasa. Traditional Tibetan architecture generally uses wooden beams and columns as load-bearing systems in the interior and mud or stone walls as load-bearing walls in the exterior. Most of the buildings do not have more than three storeys, and their plane shape is mostly rectangular. In Lhasa, "L" and "U"-shapes are the most common plane forms of traditional residential buildings, and the layout is relatively compact. In order to minimize heat loss, most spaces are closely connected with the kitchen, where the stove is located. The kitchen also assumes the function of a living room. The habits of people living around fire makes the two functional spaces of traditional Tibetan architecture overlap. Frequently used rooms, such as the living room and the main bedroom, are usually arranged in the south, while a large number of secondary bedrooms and bathrooms are placed in the north to maximize the exposure to sunlight of the frequently used rooms. On the facade, the most notable feature of Tibetan architecture is that the wall is gradually retracted upwards. The building's structure uses thicker walls at the bottom, which taper off a bit at the top. This kind of wall does not only increase the stability of the walls, but is also very effective at retaining heat. Apart from the walls, the windows above are larger than the ones below to prevent heat loss. In terms of the vertical organization of building functions, the less important functional space is usually placed at the bottom, such as rooms for livestock or storage.

In addition to the influence of natural environmental factors on the formation of traditional Tibetan architecture in Lhasa, social and human environmental factors are also very important. Influenced by the widespread influence of Tibetan Buddhism, the Buddhist room, as an important and frequently used space in Tibetan daily life, is usually arranged on the top floor of the building. In terms of architectural decoration, the exterior walls are mostly white, and white pulp is regularly sprayed on the walls. In addition to maintaining the whiteness of the wall, it also has become a part of religious customs. The parapet walls, eaves and portals of doors and windows are usually black, which plays the role of warding off evil spirits and absorbing heat. The interior decoration usually uses red, white, yellow, green, and blue patterns with religious meanings. (Fig. 1) 


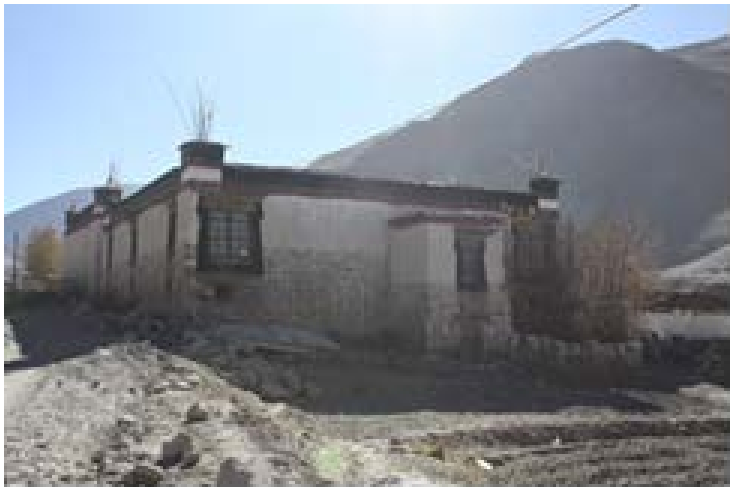

Fig. 1. Traditional buildings of Lhasa.

\subsection{The current situation of traditional architecture in Lhasa}

The existing traditional Tibetan architecture in Lhasa can be roughly divided into religious buildings, large manor buildings that are scattered throughout the Lhasa urban area, and other buildings closely arranged in Barkhor (Bakuo) Street in the old part of Lhasa's urban area, as well as temples and mansions. Apart from the temples, which still have their original religious functions, most of the other buildings have now been converted into scenic spots, or commercial or residential buildings. As a typical residential building in Tibet, the large manor buildings in Lhasa have become a hot spot for tourism development and have been mostly transformed into homestay or hotel buildings. After the government recovered the traditional Tibetan buildings in Barkhor Street, some of them were given to civilians or the descendants of the aristocrats who originally lived there, while some of them were rented out as housing. After some of these buildings were privately purchased, others became private residences or were sub-leased to merchants from all around the country. The lower floors of these buildings are used for business purposes such as operating various types of individual shops or small workshops, while the upper floors are used for accommodation. Some other buildings were transformed into hotels, teahouses, homestays, hotels, medium-sized shops and so on. The function of traditional Tibetan architecture in Lhasa has changed throughout history. In order to meet the needs of modern society, most of this architecture has become residential or commercial buildings.

\section{The significance and problems of protective reuse of traditional architecture in Lhasa}

\subsection{The significance and value of protective reuse}

The overall social level of Tibet has entered a new stage due to the continuous progress and development of the society. As the political, economic and cultural center of Tibet, Lhasa not only is responsible for promoting economic development, but should also prioritize the protecting and inheriting of Tibet's history and culture. As an important carrier of Tibetan history, culture and religious beliefs, traditional Tibetan architecture plays an important role in reflecting the development of the city. In the process of protecting traditional Tibetan architecture, reuse as a means of restoring the original function of buildings is an ideal one among many methods for protecting ancient buildings. The reuse of traditional architecture can not only discover its role as a witness to the social and economic development of each historical period, but can also extend its protection from that of the architecture itself to the surrounding environment and cities, and even that of history, culture, and people's current life. It enables people to truly gain a sense of the typical social environment and rich local historical and cultural folk traditions, and to explore a deeper comprehensive protection of the human environment and cultural characteristics.

At the same time, the rapidly developing tourism industry has become a major pillar of Lhasa's economy. In this context, we should consider the problem of how buildings can better serve the economy. With the development of cities and the tourism industry, there is an increasing demand and market for traditional buildings to be used for commercial activities. Integrating and coordinating the protective reuse and commercial reuse of traditional buildings is one of the most effective means of meeting the needs of multiple parties.

\subsection{Problems in the process of reuse}

The biggest problem in the process of the reuse of existing traditional buildings in Lhasa is the lack of professional guidance and protection. The reuse of most buildings is only to simply change their function, but this method neglects its historical and social value. At the same time, in order to meet the needs of modern life, it is extremely common for the current residents to renovate the interior space of buildings. Due to the lack of professional knowledge, the phenomenon of selfconstruction is more serious, which not only changes the spatial structure and use nature of traditional buildings, but also has great potential safety hazards. Because of users' limited awareness of protection, the ancient buildings are constantly damaged during the process of renovation, and the original traditional architectural style is destroyed.

From the user's point of view, the spatial organization form of traditional buildings may not be able to meet the needs of modern life. Many buildings have problems such as poor ventilation and lighting. In addition, since the traditional Tibetan buildings in Lhasa mostly use a wooden frame as an internal load-bearing system, the government generally does not allow private heating equipment to be added during the renovation process, resulting in the building having a low level of indoor comfort during the winter, which cannot meet basic usage and living needs.

\section{Strategies and methods of protective reuse of traditional architecture in Lhasa}




\subsection{Reuse strategy combining regional characteristics and modern demand}

The reuse of traditional buildings in Lhasa should be based on the preservation status of buildings, the surrounding environment and resources, and the functional orientation of the future development of Lhasa. On this basis, it is necessary to meet the living needs of modern residents and users and maximize the utilization of social resources. Before reusing traditional buildings, an overall evaluation and analysis of the traditional buildings and even the entire block should be carried out, and the protection and renewal should be combined during the process of reuse. For culturally significant architecture in Lhasa, such as temples and palaces, it is necessary to repair and renovate the original buildings to the greatest extent, and restore the original features of the building. The newly built buildings around them which are not in harmony with the traditional buildings should be demolished. For traditional buildings that are well preserved but are not culturally significant architecture, their original architectural functions can be retained or restored. The interior space of the buildings can be updated and rebuilt according to their functions and users, and the necessary living facilities such as a kitchen and toilet can be optimized. For the traditional buildings with relatively general preservation conditions, the renovation and transformation of the interior and exterior of the buildings can be carried out by fully combining the regional characteristics, block positioning and use requirements. The use properties and functions of the building can also be changed without destroying the traditional architectural features. The tourism facilities with distinctive features can be properly developed to meet the needs of Lhasa's new urban development orientation. Traditional buildings that have been poorly preserved can also be rebuilt according to the abovementioned reuse methods, and the buildings built in violation of the rules can be rebuilt according to the style of traditional buildings. In order to meet the requirement that the new buildings must remain consistent with the traditional style of the entire block, there should be a facade or decoration that is redesigned and reconstructed for the new buildings built in the traditional block that do not conform to its traditional features but have good architectural quality.

\subsection{Methods of protective reuse of traditional architecture}

For the reuse of traditional buildings in Lhasa, this study mainly discusses the specific methods of reuse of the traditional buildings with relatively general preservation conditions.

\subsubsection{Reusing methods based on catering space}

Transforming traditional buildings into catering space for reuse is a relatively common method in the protective reuse of traditional buildings, which also has unique advantages in Lhasa. In addition to the traditional Tibetan foods, which have become one of the indispensable experiential aspects of Tibetan tourism, the daily habit of drinking butter tea developed by Tibetans for thousands of years has also resulted in teahouses spreading throughout the streets of Lhasa and it has become an important part of urban and residential life. The "Makye Ame Tea Restaurant" located at the southeast corner of Barkhor Street in Lhasa is an example of one of the numerous renovations of dining space (Fig. 2). This restaurant was opened in a centuries-old two-storey Tibetan-style building in 1997. The facade of the building is yellow and Tibetans also call it the "Yellow House". The first floor's door is red, with traditional Tibetan patterns painted on it. The second floor's window frames are painted black according to traditional customs. The eaves of the doors and windows are adorned with traditional Tibetan decorations. The interior of the building retains the original Tibetan furniture, supplies, utensils and wall decorations, and the newly added furniture and lighting is also based on its coordination with the overall Tibetan style and features (Fig. 3). Makye Ame has become one of the must-see attractions for tourists because of its legends related to the sixth Dalai Lama, Tshangs-dbyangs-rgya-mtsho (Tsangyang Gyatso), and its effective utilization and inheritance of Tibetan culture. However, there are still some inaccurate aspects of Makye Ame. For example, although the yellow exterior wall is one of its most prominent features, based on history and due to the strict religious hierarchy, Tibetan residential buildings' walls are mostly white. Only temples, lingka (parks), palaces and high-ranking religious buildings' walls can be painted with yellow and reddish-brown. In addition, a half open space with a fourangled sloped roof is added to the top floor of the building, which is different from the flat roof of traditional Tibetan dwellings. This space was added to meet the needs of commercial sightseeing. Therefore, in the process of reusing traditional buildings, one of the key points we need to consider is whether the newly added architectural space and decorations need to follow the old social customs and order.

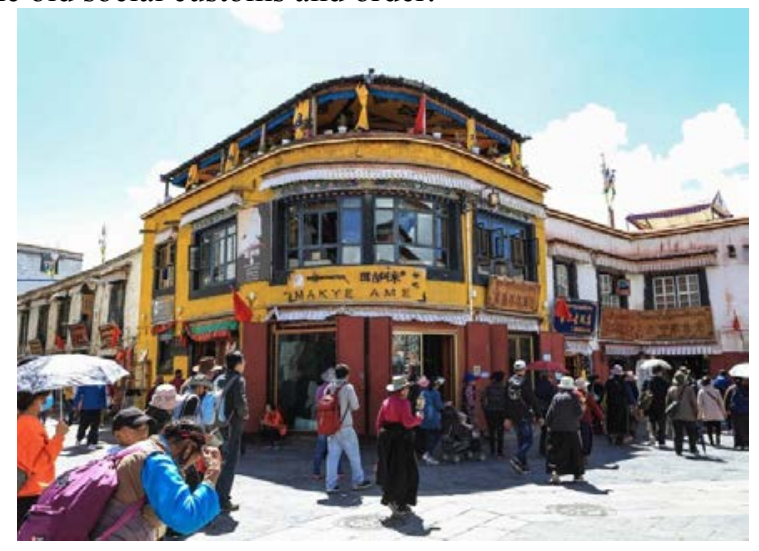

Fig. 2.Outside of makye ame tea restaurant. 


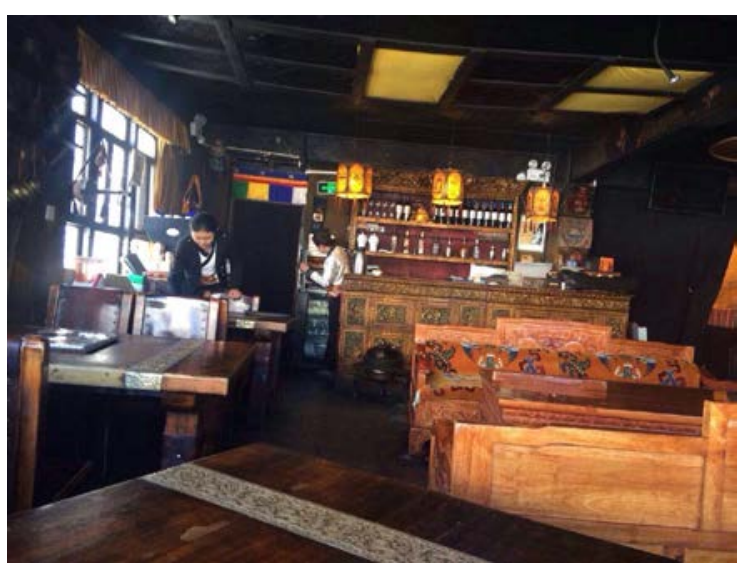

Fig. 3 Interior of makye ame tea restaurant.

\subsubsection{Reusing methods based on hotels and homestays}

The traditional architecture reuse mode based on hotels and homestays can achieve the aim of protective reuse and improve the utilization rate of traditional buildings through the reasonable renovation and repair of traditional residential buildings in Lhasa. In addition, homestays can be used to increase communication and interaction between visitors and locals so that visitors can fully understand the local history while living in a Tibetan cultural environment. There are many mansions and traditional houses in the city that have been transformed into hotels or homestays. Among them, Pandatsang, located at Barkhor South Street, is one of the hostels with many tourists. Founded in 1914, Pandatsang has an area of about $3,000 \mathrm{~m}^{2}$ and is one of the most well preserved mansions on Barkhor Street. After 1949, it also existed as post offices and courtyards inhabited by more than 70 households. In 2011, Pandatsang was transformed into a youth hostel through investment. The building structure, facade, decoration and roof are preserved so that they are basically intact. The front desk of the hotel is located on the first floor. When it was a mansion, the first floor of the building was usually used for storing goods and raising livestock, while only the second and third floors were used as residential spaces. In addition to the front desk, the colonnade of the first and second floor has been transformed into multi-person rooms for backpackers, and the main building has been converted into twin rooms. There are also problems related to reuse. Traditional Tibetan buildings have smaller spacing between columns. In the process of renovation to hotels, there are limitations in terms of the space separation and functional layout. Furthermore, because Pandatsang's original buildings did not have independent indoor toilets, partition walls had to be built for this purpose. Therefore, when the renovation was completed, Pandatsang covered a large area, but its construction area was small. It has only 40 rooms, and the largest one is $80 \mathrm{~m}^{2}$. (Fig. 4)

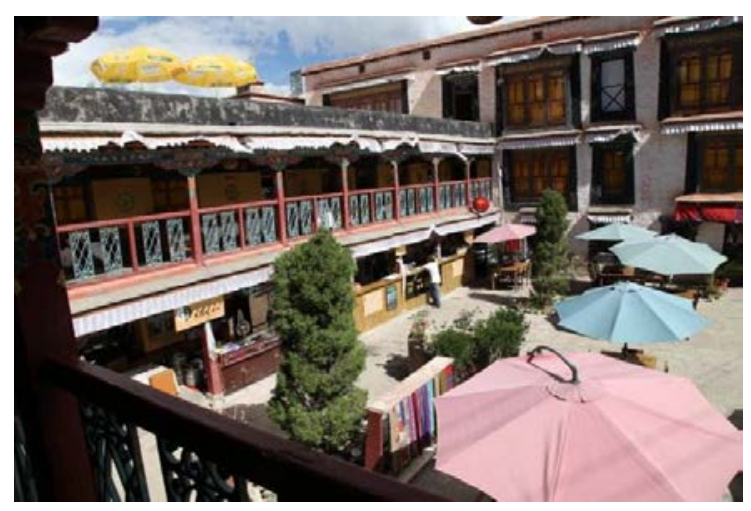

Fig. 4 Pandatsang hotel.

\subsubsection{Reusing methods based on street-facing shops}

Transformation into street-facing shops is one of the most common methods of reuse for traditional buildings in Lhasa. In Barkhor Street, except for the buildings deep in the streets, which are still used as residential space for local residents, the other buildings facing the main streets, especially the first floor, have all been transformed into small shops selling local specialties to tourists. This reuse method gives new functions to traditional buildings that are no longer used for residential space, and promotes the development of the tourism industry in the neighborhood. However, this way of reuse involves a relatively uniform business format and shop content. There is only a single transaction relationship between operators and tourists, and no deeper connection. The renovation of the buildings required an expansion of the openings of the doors and windows on the outer facade of the first floor, and the whole space was adapted for use as a business area. The second or third floors are only used as closed warehouses, so the utilization rate of the buildings is relatively low. The arrival of tourism service-oriented businesses has led to the gradual substitution of touristoriented shops for small businesses serving the local people. Tourism service-oriented businesses have become the dominant ones in Lhasa. However, due to the lack of effective control over the quality and type of business, problems that have emerged include insufficient connotations of commercial culture, underestimation of the commercial value of national culture, an excess of counterfeit and inferior commodities, and inadequate protection of commercial heritage. At the same time, merchants and enterprises engaged in tourism business services have become the "new residents" in the neighborhood by renting houses and shops. As a result, most activities in the district are guided by short-term economic interests, which reduces the residents' sense of cultural identification and responsibility. The traditional cultural atmosphere and the continuation of the cultural environment are facing difficulties. To sum up, in terms of the reusing methods based on street-facing shops, we must not blindly follow trends and mechanically copy others. The development of tourism commerce should combine Lhasa's inherent commercial culture, folk culture and various handicraft techniques to establish a benign protection mechanism for intangible cultural 
heritage, build traditional handicraft workshop areas, concentrate the local handicraft industry in the surrounding area, promote the productive protection of handicraft skills, and promote the revival of "oldfashioned" commerce. (Fig. 5)

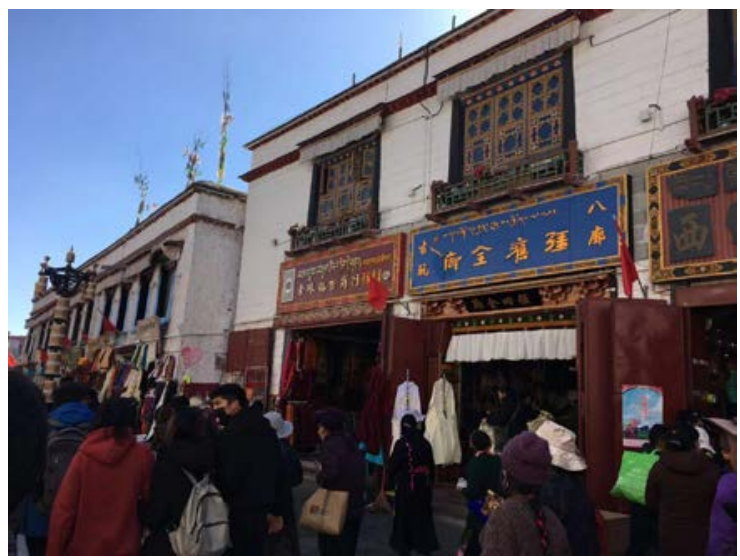

Fig. 5 Shops on barkhor street.

\section{Summary}

As an indispensable and characteristic part of the Chinese architecture system, traditional Tibetan architecture in Lhasa has a great value in relation to protection and inheritance. Compared with the single static protection mode, the dynamic reuse of buildings is a more effective means for activating architectural cultural heritage. Protective reuse emphasizes reasonable restoration, adapts to local conditions, and must not blindly follow trends and transform traditional buildings in a way that causes them to lose their regional and cultural characteristics. The protection and reuse of traditional buildings in Lhasa should also be fully combined with the orientation of Lhasa's future development. In order to promote the sustainable development of Lhasa's history and culture, and under the premise of tourism utilization, the protection of architectural heritage should be changed from simply improving living conditions to establishing the cultural identity of new and indigenous residents, as well as stimulating the enthusiasm of residents to participate in the protection of cultural heritage and encouraging them to actively pass down traditional living customs.

\section{Acknowledgments}

This work was supported by the National Key R\&D Program of China (2016YFC0700400). The authors thank the reviewers for their helpful comments and suggestions.

\section{References}

1. $\mathrm{Z} \mathrm{Xu}$. An introduction to Tibetan traditional architecture, first ed. China Architecture \& Building Press (2004)

2. S Yu. An overview of the development of Tibet architecture and decoration. Architectural Journal. 6 (2013)

3. L Huang, C Liu. The image relationship between traditional architectural space and religious culture in Tibet. Huazhong Architecture. 28, 5 (2010)

4. Y Peng, M Cao. Freely Developed Traditional Buildings in Tibet_-Primary Research on the Planning and Space Features in Tibet Traditional Buildings. Chinese and Overseas Architecture. 10 (2008)

5. J Zhuang. Traditional houses in Lhasa and architectural styles of Tibet. Planners. 2 (1995)

6. D Xu, X Dong. The social function of Tibetan folk. Journal of Sichuan University for Nationalities. 19, 1 (2010)

7. Y Chen, S Liu. The influence of Tibetan Buddhist color culture on Tibet residential architecture. Pubic Communication of Science \& Technology. 2 (2015)

8. J Zhou, T Li. Study on the Origins of Tibetan Rdzong(fort) - the Official Building. Journal of Tibet University. 23, 5 (2008) 